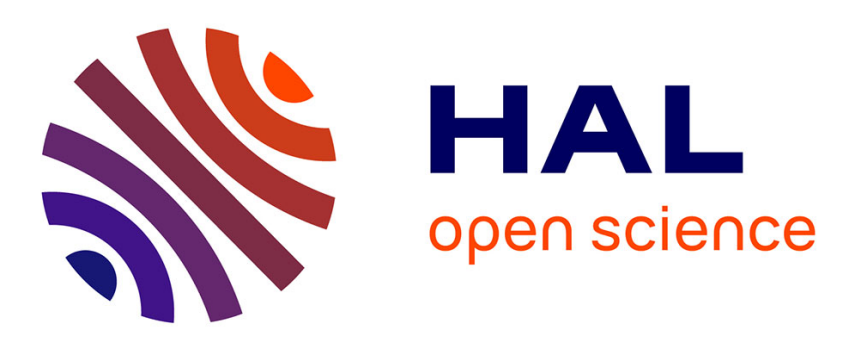

\title{
Multiple scattering of an ultrasonic shock wave in bubbly media
}

Olivier Lombard, Nicolas Viard, Valentin Leroy, Christophe Barrière

\section{To cite this version:}

Olivier Lombard, Nicolas Viard, Valentin Leroy, Christophe Barrière. Multiple scattering of an ultrasonic shock wave in bubbly media. European Physical Journal E: Soft matter and biological physics, 2018, 10.1140/epje/i2018-11624-9 . hal-02324771

\section{HAL Id: hal-02324771 \\ https://hal.science/hal-02324771}

Submitted on 24 Oct 2019

HAL is a multi-disciplinary open access archive for the deposit and dissemination of scientific research documents, whether they are published or not. The documents may come from teaching and research institutions in France or abroad, or from public or private research centers.
L'archive ouverte pluridisciplinaire HAL, est destinée au dépôt et à la diffusion de documents scientifiques de niveau recherche, publiés ou non, émanant des établissements d'enseignement et de recherche français ou étrangers, des laboratoires publics ou privés. 


\title{
Multiple scattering of an ultrasonic shock wave in bubbly media
}

\author{
Olivier Lombard, ${ }^{1}$ Nicolas Viard, ${ }^{2}$ Valentin Leroy, ${ }^{1}$ and Christophe Barrière ${ }^{2}$ \\ ${ }^{1}$ Laboratoire MSC, Université Paris-Diderot, CNRS (UMR 7057), Paris, France \\ ${ }^{2}$ Institut Langevin, Université Paris-Diderot, ESPCI, CNRS (UMR 7587), Paris, France
}

(Dated: October 24, 2019)

\begin{abstract}
This experimental study deals with the propagation of an ultrasonic shock wave in a random heterogeneous medium, constituted of identical $75-\mu \mathrm{m}$ radius bubbles, trapped in a yield-stress fluid. The fundamental frequency of the incident wave (in the $\mathrm{MHz}$ range) was much larger than the resonance frequency of bubbles $(38 \mathrm{kHz})$. A well expanded coda, resulting from the multiple scattering of the incident shock wave through the heterogeneous medium, was experimentally measured in transmission. Despite the significant amplitude of the shock wave $(90 \mathrm{kPa})$, no sign of nonlinear response of the bubbles was detected. Both the coherent and incoherent fields were successfully described by linear theories. Using a shock wave presents the advantage of characterizing the medium over a large frequency range $(1.5-15 \mathrm{MHz})$.
\end{abstract}

PACS numbers: $43.35 .+\mathrm{d}, 43.20 .+\mathrm{g}$

\section{INTRODUCTION}

Acoustic propagation in strongly scattering heterogeneous media has been widely studied for years [1-13]. In such media, the wave is multiply scattered as it encounters successively several heterogeneities. Consequently, transmitting an acoustic pulse through such a medium provides a typical signal generally showing a ballistic part at the first times of flight, followed by a multiply scattered $\operatorname{cod} a$, possibly lasting for a long time. When the positions and/or the shapes of the scatterers are random, the acoustic propagation through the medium is modeled as a random process. The transmitted wave is then the sum of a coherent [6-9] and an incoherent fields [10-13].

A simple criterion to determine whether multiple scattering occurs in a medium is $L>\ell_{s}$, where $L$ is the medium's thickness and $\ell_{s}$ its scattering mean free path, which can be though at as the average length over which the wave propagates between two scattering events [6-9]. For a random medium, without absorption, the scattering mean free path is also defined as the characteristic length of the attenuation of the coherent intensity. In presence of absorption, the characteristic length of the attenuation of the coherent intensity is the extinction mean free path $\ell_{\text {ext }}$, that takes both effects into account: scattering and absorption. When the medium is constituted of identical discrete scatterers, the scattering mean free path depends on the concentration and strength of the scatterers and the following simple formula usually applies: $\ell_{s} \approx 1 /(n \sigma)$, where $n$ is the number of scatterers per unit volume and $\sigma$ the scattering cross-section of one scatterer. It follows that strong multiple scattering can be obtained with a thick assembly (large $L$ ) of numerous (large $n$ ) and strong scatterers (large $\sigma$ ). In this case, the incoherent part of the field can be modeled as if the wave was a particule flux, where no interference occurs. The incoherent intensity obeys then to the Radiative Transfert Equation, which can be simplified to a diffusion equation, assuming the medium is statistically isotropic and using the pre-isotropic approximation, i.e.

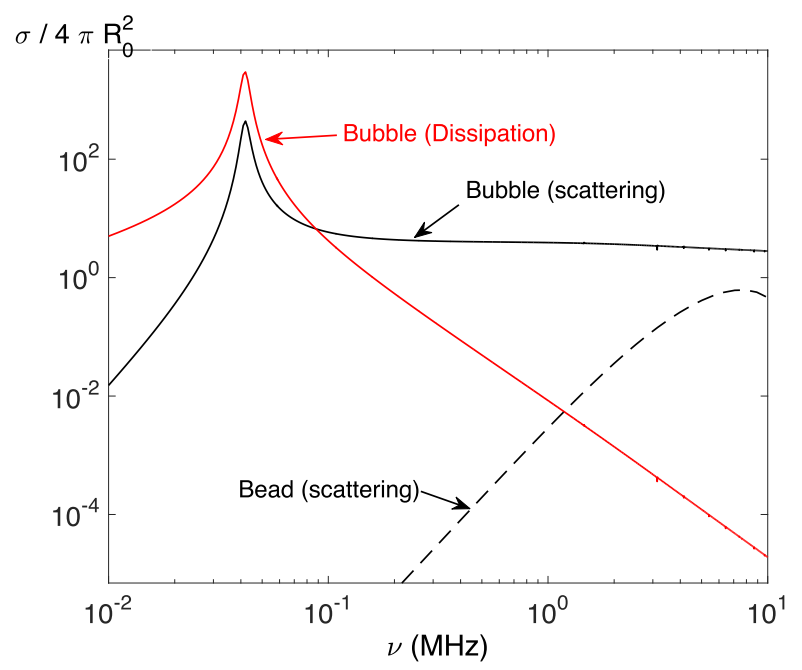

Figure 1: Scattering (black continuous line) and dissipative (red continuous line) cross-sections $\sigma$ of a nitrogen bubble of radius $R_{0}=75 \mu \mathrm{m}$ in water, versus frequency $\nu$. Black doted line: comparison with the scattering cross-section of a steel bead with the same diameter, in water. All cross-sections are normalized by $4 \pi R_{0}^{2}$, the asymptotic high frequency crosssection.

considering that the direction of the incoming wave is rapidly lost in the medium. [10-13].

Gas bubbles are good candidates as strong acoustic scatterers. A typical scattering cross-section as a function of frequency is given in figure 1, here for a $75 \mu \mathrm{m}$ radius bubbles. The bubble (black solid line) is a much stronger scatterer than a steel bead of the same size (dashed line), especially at its resonance [16], at $\nu_{0}=38 \mathrm{kHz}$. However, in the frequency range of the resonance, the absorption scattering cross-section (red line) is also large, even larger than the scattering one. It means that this regime is not favorable for the observation of coda, because most of the energy incoming on a bubble is dissipated, not scattered. Nevertheless, even 
for frequencies much higher than $\nu_{0}$, the bubble still exhibits a strong scattering cross-section, with negligible absorption.

In this paper, we study experimentally stable samples made with $75 \mu \mathrm{m}$ radius bubbles trapped in a yield-stress fluid. Those samples had a typical gas volume ratio $\phi \simeq$ $1 \%$, and they were studied over a large "high" frequency range $\left(\nu_{0} \ll 1.5 \mathrm{MHz}<\nu<15 \mathrm{MHz}\right)$, produced by ultrasonic shock waves. Using shock waves to probe these bubbly media also raises the interesting question of a possible coupling between nonlinear effects and multiple scattering.

The article is organized as follows. We first describe the experimental setup designed to study multiple scattering of ultrasonic waves in our samples. Then, we study the coherent wave transmitted by such bubbly media. The scattering mean free path $\ell_{s}$ is deduced from measurements, and compared to results provided by the ISA model. Finally, the incoherent energy of the coda is measured and compared to a diffusion equation, allowing to estimate the diffusion coefficient $D$ as a function of frequency.

\section{EXPERIMENTAL SETUP}

\section{A. Bubbly media synthesis}

Bubbly media were made with the same technique as in reference [17]: nitrogen bubbles were injected in a yield-stress fluid, with a $20-\mu \mathrm{m}$ diameter capillary driven by motorized linear stages. The yield-stress fluid was a commercial hair gel diluted with water, whose frequencydependent viscosity was determined with a rheometer: $\eta=5 / \sqrt{f / 1 \mathrm{~Hz}}$. The yield-stress was high enough to efficiently trap bubbles of radii below $100 \mu \mathrm{m}$, and it was checked that the bubbles positions were not affected by the ultrasound. Addition of $C_{6} F_{14}$ to nitrogen slowed down ripening, thus increasing the sample stability, approximately up to ten hours. Samples were contained in a $3 \mathrm{~cm}$ thickness cell (see figure 2), whose walls were as thin as possible to ensure a good transmission of ultrasounds. In practice, 14- $\mu m$ thickness Mylar membranes were used as walls. In this cell, the bubbles were injected regularly in a volume of $10 \times 10 \times 1 \mathrm{~cm}^{3}$. Then the sample was mixed to randomize the bubble positions. It was checked, by image analysis, that the thickness of the sample was not appreciably modified by this mixing: $L=1,00 \pm 0.02 \mathrm{~cm}$.

Following this whole process, we made disordered bubbly media composed of $R_{0}=75 \mu \mathrm{m}$ radius bubbles, with a gas volume fraction up to about $1 \%$. Experimental results shown in this article are almost all obtained with a sample having a gas volume ratio $\phi=0.96 \%$ (except on the inset of figure 4.b), which corresponds to a concentration of $n=5.4$ bubbles $/ \mathrm{mm}^{3}$.

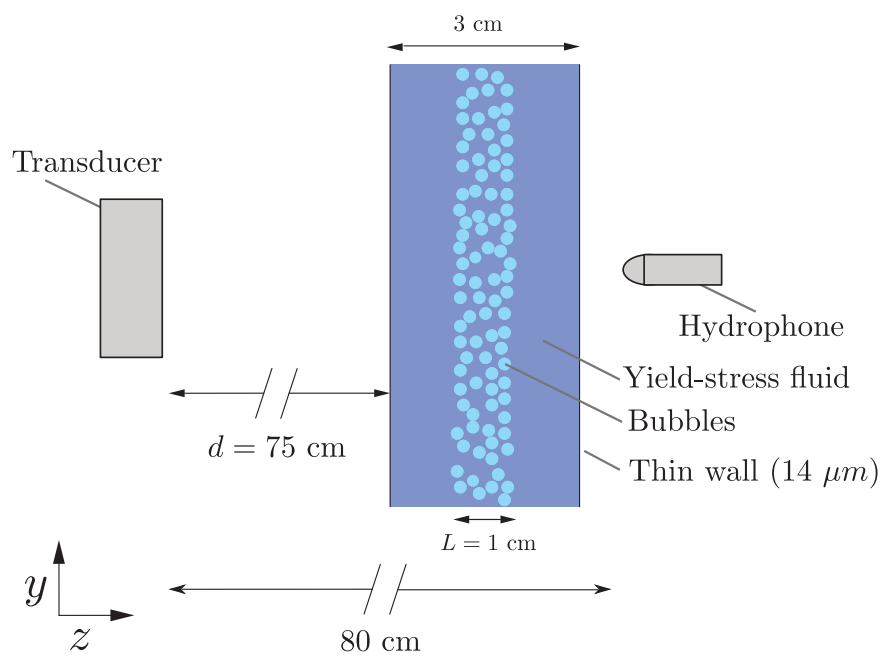

Figure 2: Experimental setup immersed in water. The transducer emits a Gaussian pulse containing 5 periods of a sinusoidal signal at a frequency $\nu_{1}=2 \mathrm{MHz}$ or $\nu_{2}=3.5 \mathrm{MHz}$. Its nonlinear propagation in water over a distance $d=75 \mathrm{~cm}$, generates a shock wave, which interacts with the bubbly sample of thickness $L=1 \mathrm{~cm}$. The hydrophone $(0.5-20 \mathrm{MHz}$ bandwidth and $0.2 \mathrm{~mm}$ lateral resolution) measures $2 \mathrm{~cm}$ behind the sample (near field), the wave transmitted through.

\section{B. Ultrasonic setup}

The experimental setup, immersed in water, is shown in figure 2. An ultrasonic transducer (with a $3.5-\mathrm{MHz}$ central frequency and a diameter $2 a=38 \mathrm{~mm}$ ) emits a pulse with a Gaussian envelope, containing 5 periods of a sinusoidal signal. This waveform was chosen to maximize the amplitude emitted by the transducer, since it lets it resonate a bit. To compensate the narrow band of such a long time signal, we perform successively two measurements at central frequencies $\nu_{1}=2 \mathrm{MHz}$ and $\nu_{2}=3.5 \mathrm{MHz}$, respectively corresponding to wavelengths $\lambda_{1}=0.75 \mathrm{~mm}$ and $\lambda_{2}=0.43 \mathrm{~mm}$ in water. The wave transmitted through the sample is measured, in the near field, with a hydrophone (having a $0.5-20 \mathrm{MHz}$ bandwidth and a $0.2-\mathrm{mm}$ lateral resolution), $2 \mathrm{~cm}$ behind the cell.

Choosing the distance $d$ between the emitter and the sample depends on both diffraction and nonlinear effects in water. Firstly, to simplify the analysis of the signals transmitted through heterogeneous samples, the incident waveform has to be stable in water along the distance corresponding to the sample thickness. Consequently, we must avoid near field diffraction effects, where interferences occur. However the sample has also to be placed not too far from the emitter, to avoid the amplitude decrease resulting from the far field lost of directivity. According to the characteristic length of diffraction $a^{2} / \lambda$, which is equal to $48 \mathrm{~cm}$ at $\nu_{1}=2 \mathrm{MHz}$ and to $84 \mathrm{~cm}$ at $\nu_{2}=3.5 \mathrm{MHz}$, the waveform is stable from a $75 \mathrm{~cm}$ to $100 \mathrm{~cm}$ distance from the transducer, in both cases. This 
point has been checked precisely by numerically solving the classical diffraction integrals [19]. So limiting diffraction effects requires to place the sample at a distance $d=75 \mathrm{~cm}$ from the transducer.

Secondly, for sufficiently large amplitudes of emission, the nonlinear propagation in water generates harmonics of the fundamental frequencies, possibly leading up to a shock wave, with a characteristic pressure discontinuity [24]. For a sinusoidal plane wave emitted in an homogeneous medium, the shock distance is: $L_{c}=1 /(k \beta M)$, where $k=2 \pi / \lambda$ is the wave-number, $\beta$ is the nonlinearity parameter of the medium, and $M=v_{0} / c_{0}$ is the acoustic Mach number, ratio of the particle velocity amplitude $v_{0}$ and the speed of sound $c_{0}$. In our experimental configuration, in water $\left(c_{0}=1500 \mathrm{~m} / \mathrm{s}\right.$ and $\beta=3.5$ ), emitting a wave with an amplitude $v_{0}=6 \mathrm{~cm} / \mathrm{s}$ (corresponding to an acoustic pressure $p_{0}=90 \mathrm{kPa}$ ) leads up to a shock distance $L_{c}=50 \mathrm{~cm}$. The calibration of our transducer with an optical interferometer has shown its capacity to emit such an amplitude [25]. Consequently, placed at the distance $d=75 \mathrm{~cm}$ from the emitter, the sample can be insonified by an ultrasonic shock wave [19].

As shown in figure $3 \mathrm{a}$, the incident signal coming on the sample is a shock wave, with the typical sharp oscillations induced by the accumulation of harmonic components (represented in figure 3.b) during the propagation in water. Using an incident shock wave enables to study the sample over a large frequency range $(1.5 \mathrm{MHz}<\nu<$ $15 \mathrm{MHz}$ ), with only two experiments driven successively at frequencies $\nu_{1}=2 \mathrm{MHz}$ and $\nu_{2}=3.5 \mathrm{MHz}$. The possibility of an interaction between nonlinear effects and multiple scattering can also be investigated.

The pressure wave $p_{i}(t)$ is measured by the hydrophone at different spatial positions $(1 \leq i \leq N)$, by moving the sample in the $(x, y)$ plane, perpendicular to the emitterreceiver axis $z$, with two motorized linear stages. We assume spatial ergodicity, implying that different realizations of the disorder can be measured at sufficiently different positions in the medium. To be certain to measure $N$ decorrelated signals, we first measure the correlation coefficient between 8 transmitted signals truncated from $t=565 \mu \mathrm{s}$, by moving the sample with a $100 \mu \mathrm{m}$ pitch. The inset of the figure 3.e shows the coefficient correlation as a function of the distance $y$ between two sample positions. We can conclude the decorrelation length of the transmitted signal is around $400 \mu \mathrm{m}$. The pressure wave $p_{i}(t)$ is measured on $N=243$ measurements, made with a $500-\mu \mathrm{m}$ pitch, larger than this decorrelation length.

\section{EXPERIMENTAL RESULTS}

\section{A. Measurement of the coherent field}

Wave $p(t)$ transmitted through a random heterogeneous medium can be theoretically modeled as the superposition of a coherent field $\langle p(t)\rangle$, i.e. the mean field
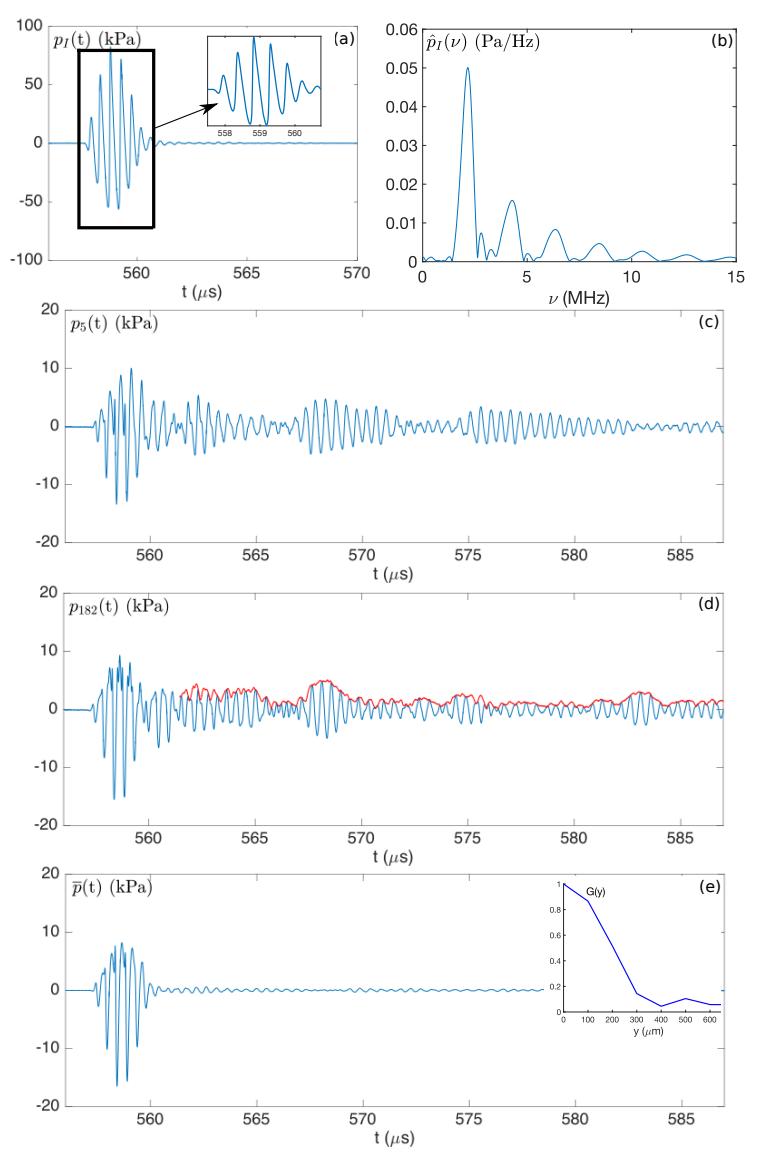

Figure 3: (a) Incident ultrasonic shock wave $p_{I}(t)$ at the fundamental frequency $\nu_{1}=2 \mathrm{MHz}$, measured in water at the distance $d=75 \mathrm{~cm}$ from the transducer. Inset : Zoom of the Incident ultrasonic shock wave. (b) Spectrum $\hat{P}_{I}(\nu)$ of the incident shock wave represented in figure 3.a.

(c) and (d) Two signals transmitted through our sample, $p_{5}(t)$ and $p_{182}(t)$, arbitrarily chosen. The envelope of the coda, calculated with a Hilbert transform, is represented in red.

(e) Coherent wave $\overline{p(t)}$, estimated by averaging $N=243$ decorrelated measurements $p_{i}(t)(1 \leq i \leq N)$. Inset : Correlation coefficient as a function of the pitch between two measured signals in the direction $y$.

averaged on an infinity of disorder realizations, and its incoherent fluctuations $\delta p(t)$, specific to each realization of the medium:

$$
p(t)=\langle p(t)\rangle+\delta p(t) .
$$

Assuming the spatial ergodicity, the coherent field $\langle p(t)\rangle$ is experimentally estimated by the average $\overline{p(t)}$ of the $N=243$ measurements $p_{i}(t)$, acquired for different positions of the sample:

$$
\overline{p(t)}=\frac{1}{N} \sum_{i=1}^{N} p_{i}(t) .
$$

Figures $3 \mathrm{c}$ and $3 \mathrm{~d}$ show two signals $p_{5}(t)$ and $p_{182}(t)$, arbitrarily chosen, produced by the incident shock wave 
$p_{I}(t)$ (fundamental frequency $\nu_{1}=2 \mathrm{MHz}$ ), represented in figure $3 \mathrm{a}$, through the sample. They both exhibit a very well-developed coda, spreading over more than $50 \mu \mathrm{s}$, whereas the incident wave goes down after a time $T \simeq$ $3 \mu \mathrm{s}$. Note that shorter coda waves had already been reported in bubbly media $[17,20,21]$.

The coherent wave $\overline{p(t)}$, shown in figure $3 \mathrm{e}$, still looks like a shock wave, but its waveform is quite different from that of the incident shock wave. Indeed, their phases seem to be opposite, and small secondary short peaks (high frequency contents) are visible in the coherent wave, suggesting a possible nonlinear interaction with the medium. Modeling the propagation through the sample will clarify this point.

\section{B. Comparison with the linear ISA model}

In this section, we apply the well-known linear "Independent Scattering Approximation" model to our experimental configuration. Our goals are to determine the scattering mean free path $\ell_{s}$, and to bring out possible differences with our experiments, due to nonlinear multiple scattering. If $\vec{r}(x, y, z)$ denotes the position, let us consider a sinusoidal plane wave $e^{j\left(\overrightarrow{k_{0}} \cdot \vec{r}-2 \pi \nu_{0} t\right)}$, of frequency $\nu_{0}$ and wave-number $k_{0}=\frac{2 \pi \nu_{0}}{c_{0}}$, propagating in water. The time dependence $e^{-j 2 \pi \nu_{0} t}$ will be omitted in the following calculation, because it plays no part.

When this plane wave is scattered by a bubble (of radius $R_{0}$ ), the acoustic field becomes:

$$
e^{j \vec{k}_{0} \cdot \vec{r}}+f(\theta) \frac{e^{j k_{0} r}}{r}
$$

where $f$ is the scattering function of the bubble, depending on the angle $\theta$ between the incident and scattered wave-numbers. $f$ is calculated following the reference [23].

The "Independent Scattering Approximation" consists in neglecting scattering loops and position correlations between scatterers. Within this framework, the coherent field propagates with an effective wave-number $k_{\text {eff }}$, given by $[1,6]$ :

$$
k_{\mathrm{eff}}^{2} \simeq k_{0}^{2}+4 \pi n f_{0}
$$

where $f_{0}=f(\theta=0)$ is the forward scattered wave.

Following this approximation, the acoustic transmission factor $T$ of a multiply scattering medium with a thickness $L$ is:

$$
T(L)=e^{-\frac{L}{2 \ell_{s}}} e^{j \frac{2 \pi \nu}{v_{\phi}} L}
$$

where $\ell_{s}=1 /\left[2 \Im\left(k_{\text {eff }}\right)\right]$ is the scattering mean free path, i.e. the characteristic distance of attenuation of the coherent intensity (already mentioned in the introduction),

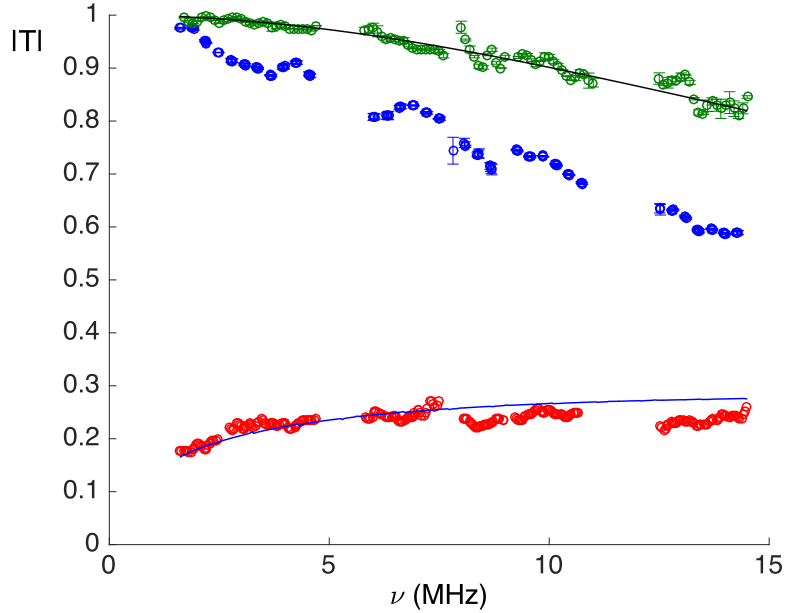

(a)

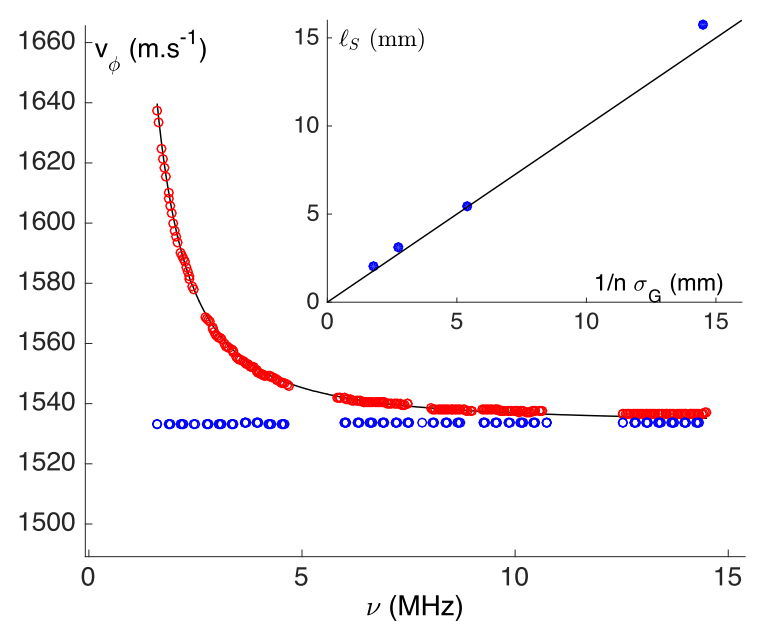

(b)

Figure 4: (a) Acoustic transmission factor modulus $|T|$ versus frequency $\nu$, of the Mylar walls alone in water (green points), of the yield-stress-fluid (blue points) and of the bubbly media (red points). For the Mylar walls, measurements are compared to the linear transmission through two $14 \mu \mathrm{m}$ layers (mass density $\rho_{M}=1400 \mathrm{~kg} / \mathrm{m}^{3}$ and speed of sound $\left.c_{M}=2550 \mathrm{~m} / \mathrm{s}\right)$, represented by a solid black line. Measurements in the bubbly medium are compared to the ISA model (solid blue line).

(b) Phase velocity $v_{\phi}$ in the cell with (red points) and without (blue points) bubbles, versus frequency $\nu$. Again, measurements in the bubbly medium are compared to the ISA model (solid black line). Inset: scattering mean free path $\ell_{s}$ measured at $2 \mathrm{MHz}$ in samples having different gas concentrations $n$ (blue points) compared to ISA prediction.

and $v_{\phi}=2 \pi \nu /\left[\Re\left(k_{\text {eff }}\right)\right]$ is the phase velocity of the coherent field.

Figure 4 shows the modulus of the acoustic transmission factor $|T|$ and the phase velocity $v_{\phi}=2 \pi \nu L / \arg T$, versus frequency $\nu$.

In figure 4.a, the transmission factor of the Mylar walls 
in water is compared to the linear transmission through two $14 \mu \mathrm{m}$ layers, with a mass density $\rho_{M}=1400 \mathrm{~kg} / \mathrm{m}^{3}$, and in which sounds propagates at $c_{M}=2550 \mathrm{~m} / \mathrm{s}$. Viscous losses due to the yield-stress-fluid are also quantified from experimental data. Finally, the experimental transmission factor of the bubbly medium, deconvolved from Mylar walls effects and viscous losses, is compared to the results predicted by the linear ISA model, with a general good agreement. At highest frequencies (approximately between 13 and $15 \mathrm{MHz}$ ), measurements come from the fourth harmonic component of the $3.5-\mathrm{MHz}$ frequency shock wave, giving a poor signal-to-noise ratio, that generates small discrepancies with the model.

Assuming the exponential decay predicted by the ISA model, the scattering mean free path can be determined from $|T|: \ell_{s}=-L /(2 \ln |T|)$. In our sample described in section II A, $\ell_{s}$ is equal to $2.5 \mathrm{~mm}$ at $2 \mathrm{MHz}$, and to $3 \mathrm{~mm}$ at $3.5 \mathrm{MHz}$. Then it increases slowly up to $3.5 \mathrm{~mm}$ at $15 \mathrm{MHz}$.

In figure 4.b, the phase velocity measured in the bubbly medium is in very good agreement with the velocity provided by the ISA model. Due to multiple scattering, the propagation of the coherent wave is strongly dispersive up to approximately $5 \mathrm{MHz}$, whereas no dispersion is noticed in the cell without bubbles.

Measurements were also made through four samples having different gas concentrations. In the inset of figure 4.b, measured scattering mean free paths at $2 \mathrm{MHz}$ are in good agreement with those predicted by the ISA model.

The good description of our measurements by the ISA model indicates that the acoustic propagation remains strictly linear in our experiments, contrary to what the shape of the coherent wave may suggest. To bring another proof of the linear behavior of our sample, we have compared, in figure 5 , the transmission factor $|T|$ of the fundamental frequency band centered at $\nu_{2}=3.5 \mathrm{MHz}$ and the transmission factor of the second harmonic frequency band centered at $4 \mathrm{MHz}$, resulting from the nonlinear propagation of the wave at the fundamental frequency $\nu_{1}=2 \mathrm{MHz}$. Although the amplitude of the second harmonic $2 \nu_{1}$ is approximately half of that of the fundamental signal at $\nu_{2}$, the two acoustic transmission factors are identical, confirming the linearity of the process.

Thus, the differences between the incident and the coherent waveforms can be ascribed to the effect of dispersion induced by multiple scattering. Figure 6.a shows the signal obtained by applying the ISA model to the incident shock wave $p_{I}(t)$ represented in figure 3.a. The resulting waveform is very close to that of the coherent wave $\overline{p(t)}$, represented in figure 3.b. Figure 6 .b also represents the signal obtained by applying only the modulus $|T|$ of the transmission factor given by the ISA model to $p_{I}(t)$, with the constant argument of the transmission factor $\arg (T)$, measured in the yield-stress-fluid. Then the small secondary short peaks noticed in the coherent wave at the end of section (III A) are clearly due to the dispersion of the phase velocity $v_{\phi}$. As a conclusion, in

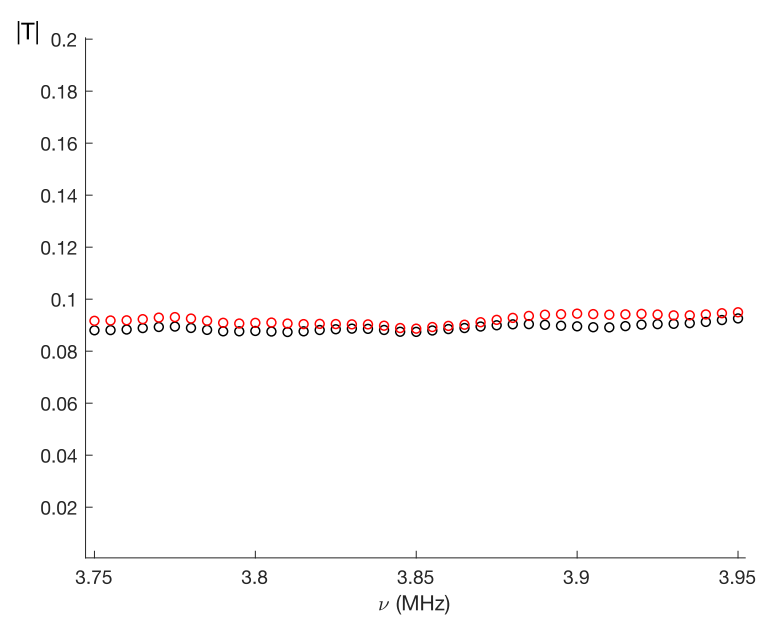

Figure 5: Comparison, over the 3.75-4 MHz range, between the transmission factors $|T|$ of the fundamental frequency band centered at $\nu_{2}=3.5 \mathrm{MHz}$ (in red), and the second harmonic frequency band centered at $4 \mathrm{MHz}$ (in blue), resulting from the nonlinear propagation of the wave at the fundamental frequency $\nu_{1}=2 \mathrm{MHz}$.

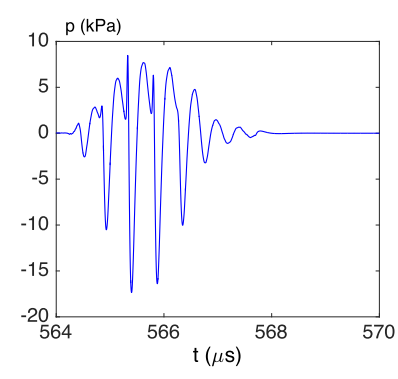

(a)

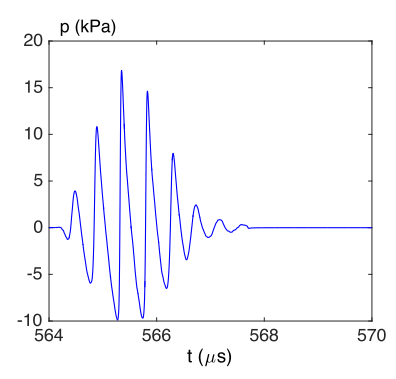

(b)
Figure 6: (a) Pressure obtained by applying the whole ISA model to the incident shock wave $p_{I}(t)$.

(b) Pressure obtained by applying only the modulus $|T|$ of the transmission factor given by the ISA model to $p_{I}(t)$, and the constant argument $\arg (T)$ measured in the yield-stress fluid.

our bubbly sample, multiple scattering dominates over nonlinear effects over the frequency range investigated.

\section{Incoherent field}

In an infinite scattering medium, when the propagation distance is larger than the scattering mean free path $\ell_{s}$, th and when the diffused energy becomes quasi-isotropic, the local energy density $W$ of the incoherent field obeys to a diffusion equation governed by a coefficient $D\left[10^{-}\right.$ $13]$ :

$$
\frac{\partial W}{\partial t}=D \Delta W
$$

In a medium consisting of identical scatterers, the characteristic distance of this diffusion process is the trans- 
port mean free path:

$$
\ell^{*}=\frac{\ell_{s}}{1-\langle\cos \rangle},
$$

where the mean cosine of the scattering angle $\theta$ is:

$$
\langle\cos \rangle=\frac{\int_{0}^{\pi}|f(\theta)|^{2} \cos (\theta) \sin (\theta) \mathrm{d} \theta}{\int_{0}^{\pi}|f(\theta)|^{2} \sin (\theta) \mathrm{d} \theta} .
$$

Transport mean free path $\ell^{*}$ can be interpreted as the distance required for a plane wave to lose the memory of its initial direction in the scattering medium. This distance depends both on the number of scatterers per volume unit $n$, and on their scattering function $f$. For an isotropically scattering heterogeneity, $\langle\cos \rangle=0$ and $\ell^{*}=\ell_{s}$. At the opposite, for a heterogeneity scattering strongly forward, $\langle\cos \rangle \rightarrow 1$ and then $\ell^{*} \rightarrow \infty$.

Using the relation between $\ell^{*}$ and $\ell_{s}$ given by the equations (7) and (8), we can deduce $\ell^{*}$ from the measurements of $\ell_{s}$ made in our experiments (see section (III B)): it increases from $2 \mathrm{~mm}$ at $2 \mathrm{MHz}$ to $7 \mathrm{~mm}$ at $10.5 \mathrm{MHz}$. So the diffusive regime is effective in our sample, since its thickness $L=10 \mathrm{~mm}$ is always larger than $\ell^{*}$.

Transport mean free path $\ell^{*}$ is also related to the diffusion coefficient $D$ and to the transport speed of the incoherent energy $c$ by:

$$
D=\frac{\ell^{*} c}{3}
$$

As described in the reference [11], the finite thickness $L$ of our samples can be taken into account by adding boundary conditions to equation (6), leading to a modal decomposition of $W$ :

$$
W(z, t)=\sum_{m=1}^{\infty} A_{m} \sin \left(\frac{m \pi\left(z+\frac{2 \ell^{*}}{3}\right)}{L+\frac{4 \ell^{*}}{3}}\right) \mathrm{e}^{-D\left(\frac{m \pi}{L+\frac{4 \ell^{*}}{3}}\right)^{2} t} .
$$

Each mode $m$ exponentially decreases with a characteristic time inversely proportional to $\mathrm{m}^{2}$ :

$$
\tau_{D_{m}}=\frac{\left(L+4 \ell^{*} / 3\right)^{2}}{m^{2} \pi^{2} D} .
$$

Thus, for sufficiently long times, the first diffusive mode dominates all the others. In particular, the second diffusive mode becomes negligible before the first one when $t>\frac{\tau_{D_{1}}}{3}$.

Experimentally, we can measure the acoustic intensity of the incoherent wave, which is the average value of the Poynting vector $\vec{J}$ (density of the power flux of the wave: $\vec{J}=-D \vec{\nabla} W$ ) over the time length $T \simeq 3 \mu$ s of the incident wave. As $\vec{J}$ has the same time dependence as $W$, measurements can be compared to theoretical results given by equation (10).

The $N$ measurements $p_{i}(t)$ are first frequentially filtered, on 6 successive $2-\mathrm{MHz}$ frequency bands, centered on frequencies: $\nu_{1}=2 \mathrm{MHz}, \nu_{2}=3.5 \mathrm{MHz}, 2 \nu_{1}=4 \mathrm{MHz}$, $3 \nu_{1}=6 \mathrm{MHz}, 2 \nu_{2}=7 \mathrm{MHz}$, and $3 \nu_{2}=10.5 \mathrm{MHz}$.

Then, the envelopes of the signals $p_{i, \nu}(t)$ resulting from this filtering, are calculated with a Hilbert transform (HT). In each 2-MHz band, the mean incoherent intensity is obtained by averaging the envelopes of the $N$ measurements. Finally, the estimator of the mean incoherent intensity is normalized by the intensity $I_{0, \nu}$ of the incident wave filtered in a same way:

$$
\frac{\left\langle I_{\nu}(t)\right\rangle}{I_{0, \nu}}=\frac{\frac{1}{N} \sum_{i=1}^{N}\left|\operatorname{HT}\left(p_{i, \nu}(t)\right)\right|^{2}}{\frac{1}{T} \int_{0}^{T} p_{I, \nu}^{2}(t) d t} .
$$

Figure 7 shows the mean incoherent intensities measured around the frequencies $\nu_{2}=3.5 \mathrm{MHz}$ and $2 \nu_{2}=$ $7 \mathrm{MHz}$, versus time. At $7 \mathrm{MHz}, \frac{\left\langle I_{\nu}(t)\right\rangle}{I_{0, \nu}}$ tends towards a constant value, giving the noise level. For times respecting the condition $t>\frac{\tau_{D_{1}}}{3}$, experimental results are fitted with exponential functions. A measurement of the characteristic time of the first diffusive mode $\tau_{D_{1}}$ is directly given by the slope of the fit.

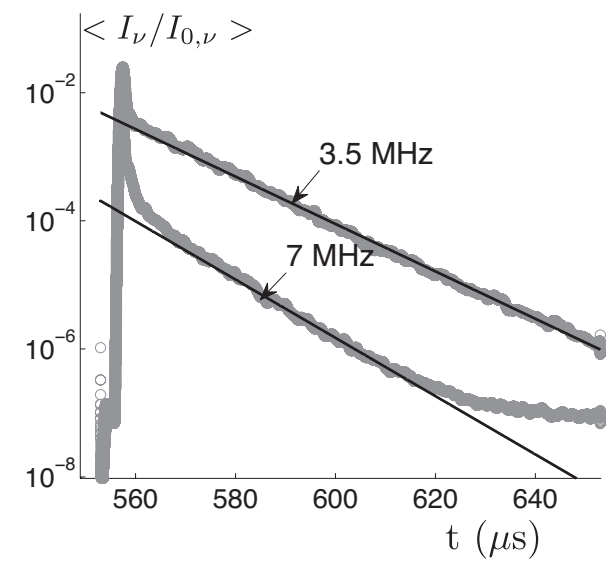

Figure 7: Mean incoherent intensities measured around frequencies $\nu_{2}=3.5 \mathrm{MHz}$ and $2 \nu_{2}=7 \mathrm{MHz}$, versus time. Experimental results are fitted with an exponential function $e^{-t / \tau_{D_{1}}}$.

In figure 8 , theoretical results provided by equation (11) are compared to experiments, assuming that the speed of the incoherent energy is that of the yield-stressfluid: $c=1530 \mathrm{~m} / \mathrm{s}$. The error bars are calculated from the $95 \%$ confidence interval of the exponential fits. As for the coherent wave studied in section (IIIB), measurements are in good agreement with a linear theoretical model leading to the same conclusion: no nonlinear effect occurs in the sample, where the propagation is dominated by the multiple scattering.

The diffusion coefficient $D$ is deduced from the measurement of $\tau_{D_{1}}$. It increases slowly from $D=1 \mathrm{~m}^{2} / \mathrm{s}$ at $2 \mathrm{MHz}$ to $D=3.5 \mathrm{~m}^{2} / \mathrm{s}$ at $10.5 \mathrm{MHz}$. 


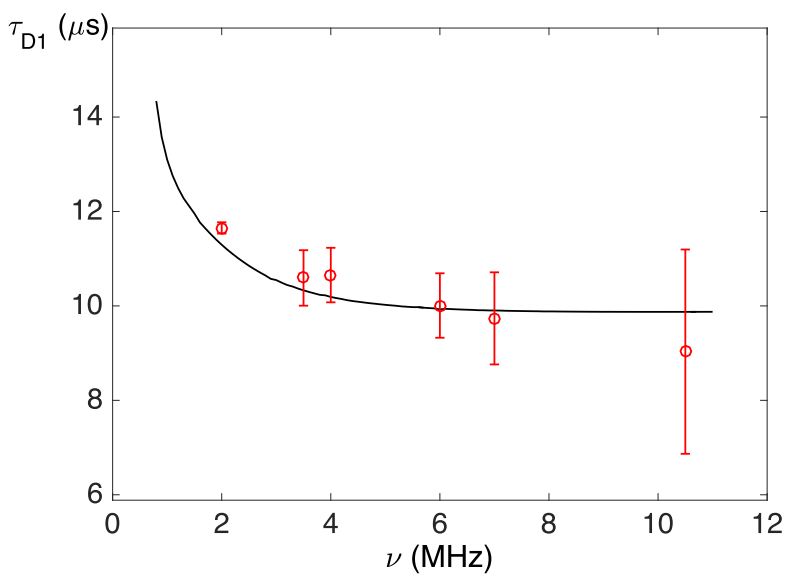

Figure 8: Measurements of the characteristic time $\tau_{D_{1}}$ of the first diffusive mode (red points), compared to theoretical results provided by equation (11) (solid black line). The incoherent energy is assumed to be transported at the speed of sound of the yield-stress-fluid ( $c=1530 \mathrm{~m} / \mathrm{s})$, whatever the frequency.

\section{CONCLUSION}

When an acoustic wave propagates in a stable bubbly medium, at frequencies higher than the Minnaert resonance of the bubbles, a very well developped coda is observed. In such case, the wave is the superposition of a coherent field and incoherent fluctuations resulting from the disorder of the bubbles. Using an incident shock wave, we have measured the scattering mean free path $\ell_{s}$, the coherent phase velocity $v_{\phi}$ and the diffusion coefficient $D$, in our samples, over a broad frequency range. The coherent field propagation is well described by the Independant Scattering Approximation, and the intensity of the incoherent field by a diffusion equation. Following these results, the time evolution of our bubbly samples will be investigated in the future, using diffusive wave spectroscopy $[14,15]$.

From our experiments, we can also conclude there is no coupling between nonlinear and multiple scattering effects, when a bubbly medium is excited at frequencies much higher than the Minnaert resonance of its bubbles. Indeed, all the harmonic components of the incident shock wave propagate independently. These results are coherent with litterature, where nonlinear effects were already observed in bubbly media, excited at frequencies smaller than the Minnaert resonance [26-28]. However, multiple scattering effects are small at these frequencies. Finally, multiple scattering effects can be observed in nonlinear regime only if bubbles are excited around Minnaert frequency $[29,30]$.

We acknowledge funding support from the Agence Nationale de la Recherche (ANR-11BS09-007-03), project DiAMAN.
[1] A. Ishimaru : Wave Propagation and Scattering in Random Media. New York : Academic Press (1978).

[2] A. Lagendijk, B. A. Van Tiggelen, Resonant Multiple Scattering of light. Physics Reports, 270143 (1996).

[3] S. M. Rytov, Y. A. Kravtsov, V. I. Tatarskii, Principles of Statistical Radiophysics 4, Propagation Through Random Media. Springer Verlag, Belin Heidelbert (1989).

[4] S. Skypetrov, B. Van Tiggelen, Wave scattering in Complex Media From Theory to Applications, NATO science Series, Vol 107, Kluwer, Dordrecht (2003).

[5] A. Derode, A. Tourin, M. Fink, Phys. Rev. E 64, 036605 (2001).

[6] L. Foldy, Phys. Rev. 67, 107 (1945).

[7] P. C. Watermann, R. Truel, J. Math. Phys. 2, 512 (1961).

[8] C. M. Linton, P. A. Martin, J. Acoust. Soc. Am. 117, 3413 (2005).

[9] A. Derode, V. Mamou, A. Tourin, Phys. Rev. E. 74 (2006).

[10] J. H. Page, H. P. Schriemer, I. P. Jones, P. Sheng, D. A. Weitz. Physica A 241, 64 (1997).

[11] J. H. Page, H. P. Schriemer, A. E. Baily, D. A. Weitz, Phys. Rev. E 52, 3106, (1995).

[12] A. Aubry, A. Derode, Phys. Rev. E 75, 026602 (2007).

[13] B. Tallon, T. Brunet, J. H. Page, Phys. Rev. Lett. 119, 164301 (2017).

[14] V. Leroy, A. Derode, Phys. Rev. E, 77, 036602 (2008).
[15] M. L. Cowan, I. P. Jones, J. H. Page, D. A. Weitz, Phys. Rev. E, 65, 066605 (2002).

[16] M. Minnaert, Phil. Mag, 235-248 (1933).

[17] V. Leroy, A. Strybulevych, J. H. Page, J. Acoust. Soc. Am, 123 (2008).

[18] P. Coussot, J. Non-Newtonian Fluid Mech., 211, 31-49, (2014)

[19] N. Viard, B. Giammarinaro, A. Derode, C. Barrière, Phys. Rev. E, 88, 023201 (2013).

[20] F. Van Der Biest, Diffusion multiple et renversement du temps ultrasonore dans des milieux périodiques et désordonnés. Thèse de doctorat de l'Université Paris Diderot (2005).

[21] I. S. Kol'tsova, L. O. Krynskii, I. G. Mikhailov, I. E. Pokrovskaya, Akust. Zh. 25, 725-731 (1979).

[22] T. G. Leighton, The Acoustic bubble, Academic Press.

[23] J. R. Allegra, S. A. Hawley, J. Acoust. Soc. Am, 51, 1545 (1972).

[24] M. F. Hamilton, D. T. Blackstock, NonLinear Acoustics. Academic, New York (1998).

[25] C. Barrière, D. Royer, Appl. Phys. Lett., 79(6), 878-880 (2001).

[26] E.A. Zabolotskaya, Soviet Physic Acoustic 21(6), 569-571 (1976).

[27] J. Wu, Z. Zhu, The Journal of the Acoustical Society of America, 89(6) (1991). 
[28] J. Wu, J. Tong, Ultrasound in medicine and Biology, 24(1), 153-159 (1997).

[29] O. Lombard, C. Barrière, V. Leroy, Europhysics Letters, 112,24002 (2015).
[30] O. Lombard, C. Barrière, V. Leroy, Ultrasonics, 78, 110114 (2017). 